\title{
The Challenge of Childhood Diabetes
}

\author{
Abdullah M Nasrat ${ }^{1^{*}}$, Salwa AM Nasrat ${ }^{2}$, Randa M Nasrat ${ }^{3}$ and Mohammad M Nasrat ${ }^{3}$ \\ ${ }^{1}$ Department of Surgery, Balghsoon Clinic, Jeddah, KSA \\ ${ }^{2}$ Department of Physical Therapy, Cardiac Surgery Academy, Cairo, Egypt \\ ${ }^{3}$ Department of Internal Medicine, Helwan General Hospital, Helwan, Egypt \\ *Corresponding author: Abdullah M Nasrat, Department of Surgery, Balghsoon Clinic, Jeddah, PO Box 5261, Jed. 21573, KSA, Tel: + 966 (012) 667 3645; Fax: + 966 \\ (012) 667 3645; E-mail: abdullahalnasrat@yahoo.com
}

Rec date: June 16, 2015 Acc date: July 16, 2015 Pub date: July 22, 2015

Copyright: (C) 2015 Nasrat AM, et al. This is an open-access article distributed under the terms of the Creative Commons Attribution License, which permits unrestricted use, distribution, and reproduction in any medium, provided the original author and source are credited.

\begin{abstract}
The study aimed at demonstration of a correlation between the challenge of Helicobacter pylori prevalence worldwide and the challenging spread of childhood diabetes in the world. The rising frequency of childhood diabetes mellitus in the world should not be taken in separate consideration from the worldwide dramatic spread of adult diabetes mellitus (DM). The correlation between diabetes and the increased incidence of obesity in children should be also considered.
\end{abstract}

$H$. pylori were suggested as one of the environmental reasons that could be directly related to the problem of childhood DM. The challenge lies mainly in the emergence of drastic resistant $H$. pylori strains due to the antibiotic violence against the stomach bacterium; these strains can travel from parents during early childhood to kids leading to a state of biological stress that could lead to stress diabetes; interestingly, children maintain the same strain genotype of $H$. pylori lifelong even they move to a different environment. The current eradication treatments of $H$. pylori have shown a lot of controversy; it would be a plea to cost the child's delicate structure the drastic side-effects of repeated antibiotic eradication therapy upon detection of $H$. pylori each time.

The study included 7 children aged between 6-9 years old with an early onset of diabetes mellitus. Children were investigated together with their parents for the existence of $H$. pylori using specific tests. Colon-care and colon-clear with natural purge and bio-organic acids were employed for $H$. pylori eradication for children and parents. All children and their parents were found positive for $H$. pylori; all children became free of $H$. pylori strains after colonclear while parents of three families needed revision of colon clear in order to complete eradication of $H$. pylori. The diabetic condition was successfully recovered in 9 children and they were able to quit therapy and maintain normal blood sugar values; one child aged 8 years old had to continue on insulin in order to maintain normal blood sugar level.

It was concluded that the challenge of childhood diabetes could be simply part of the $\mathrm{H}$. pylori-related worldwide dramatic spread of DM. Natural colon-clear should be considered as safe and effective measure for eradication of the abnormal-habitat colonic $H$. pylori strains. Revision of the guide lines of the newly discovered childhood diabetes should be considered.

Keywords: H. pylori; Childhood diabetes; Hyperglycemia

\section{Introduction}

The frequency of ketoacidosis at the onset of childhood in the world particularly in developing countries is significant. Prevention of diabetic ketoacidosis and control of its rising frequency should be a healthcare target [1]. The epidemic of childhood diabetes is a worldwide challenge that could be directly related to the world's challenging epidemic of childhood obesity or it is simply part of the dramatic spread of adulthood diabetes worldwide [2-4].

Similar to adult diabetes mellitus (DM), Helicobacter pylori could constitute a major environmental reason that could be directly related to the challenge of childhood diabetes. It has been referred to a possible relation between $H$. pylori and diabetes mellitus in children in some studies, while this relation has been denied by other reports [5,6]. H. pylori has got an extreme widespread world prevalence; if the organism does not exist in the stomach of all the population in developing countries, the latest knowledge is that $80-90 \%$ of adults are estimated to be affected with $H$. pylori in these countries [7]. The flare up of a lot of worldwide medical problems related to $H$. pylori should attract the attention towards the possibility that the stomach bacterium could be responsible in part for the problem of DM in children. This possibility deserves to be seriously considered particularly if autoimmune pancreatitis comes among the medical dilemmas related to $H$. pylori. Moreover, this suggestion is further supported by the fact that tonsils and adenoids are recently discovered as secondary reservoirs for $H$. pylori in children [8,9]. In addition, the diabetic condition was successfully corrected in adults by mere eradication of $H$. pylori. Different reports in literature have confirmed the association of adenotonsillar hypertrophy in children with the existence of cytotoxin-associated gene A (cagA) positive $H$. pylori strains, and emphasized that cagA of $H$. pylori encodes a highly immunogenic and virulence-associated protein; the presence of this 
virulent gene in the body could affect the clinical outcome in many children $[10,11]$.

\section{Aim \\ Demonstration of a possible relation between the worldwide spreading prevalence of $H$. pylori and the rising challenge of childhood diabetes in the world. \\ Design and Setting \\ A multiple-case clinical study which was done in Balghsoon Clinics in Jeddah/Saudi Arabia during the period May 2012 to October 2013. The protocol of the study was approved and the study followed the research committee ethics of Balghsoon Clinics.}

\section{Patients and Methods}

The study included 7 children with average body built and an average age range of 6-9 years old who were discovered with an early onset of hyperglycemia; wasting of weight and diuresis were observed on them and hence blood sugar level was tested and found above 290 $\mathrm{mg} / \mathrm{dl}$, the glycated hemoglobin (HbAIc) was above 11.5; confirming the onset of diabetes. Appearance and spontaneous disappearance of the diabetic condition in 4 children of them (57.1\%) was a motive to attract the attention towards the possibility of a potential condition causing temporary insult to the pancreas that recovers spontaneously. An influence of $H$. pylori was suggested; accordingly existence of $H$. pylori was tested in all children and their parents by employing specific tests (urea breath test and $H$. pylori fecal antigen) $[9,10]$.

Immediate natural therapy for $H$. pylori eradication that consisted of colon clear employing the natural senna leaves purge together with colon care using bio-organic acids (lactic and acetic) were employed for all children and their parents.

Urea breath test was available from Helicap Co., Sweden with Batch No. HCO1150108-E10, while the $H$. pylori fecal antigen test was obtained from Acon Laboratory, USA, Batch No. HP8040008.

\section{Results}

All children and their parents were found positive for $H$. pylori existence; they became free of $H$. pylori strains in the stomach and the colon after the natural therapy as confirmed by specific sensitive tests (urea breath test and $H$. pylori fecal antigen) except parents of three families who needed revision of colon clear in order to achieve complete eradication of $H$. pylori. The diabetic condition has been successfully corrected in 6 children $(85.7 \%)$ without any insulin therapy with a blood sugar range of 100-126 mg/dl (HbAIc 5-6), while one diabetic child (14.3\%) failed to respond to the natural therapy. Interestingly; the four children who were showing appearance and spontaneous disappearance of hyperglycemia before the study demonstrated dramatic response to the natural therapy. The diet of children and their bowel motion habits were watched, meanwhile their medical condition was followed up for 18 months. Recurrence of the diabetic condition within 3-5 months occurred in 3 children (42.9\%) due to misbehavior in food intake or recurrence of abnormal behavior $H$. pylori strains from one the parents; the diabetic condition was corrected in $2(28.6 \%)$ of them.

The results of this study was compared with records of other 6 children of rather similar body built and rather similar age range (6-10 years) who newly developed diabetes mellitus with blood sugar range between 260-270 mg/dl (HbAIc 10.5-11); their parents preferred to put them on insulin therapy. Their diabetic condition remained inadequately controlled in spite of regular assessment of therapy and carefulness about their food habits.

\section{Ethical Considerations}

An informed signed consent was taken from all parents; children were allowed to lead their routine style of life except strict follow up of diabetic diet and extreme restriction of outside-home food intake. Parents were free to make their children quit the study at any time whenever they feel inconvenience towards the natural therapy.

\section{Discussion}

The clinical presentation of type 1 childhood DM was studied by some researchers in developing countries, it was emphasized that the clinical picture of type 1 childhood diabetes in developing countries seems to differ from that in developed countries; [12] the difference in $H$. pylori prevalence in those countries might be a reason. The world literature does not have sufficient explanation for the rising burden of diabetes in children [2,3]. In the same way, pediatricians do not possess good reasons for the children's diabetic phenomena in developing countries [13]. The world literature refers with concern to the association and role of $H$. pylori in the adult metabolic syndrome. It was also shown that prevention of the metabolic syndrome was achieved by $H$. pylori eradication in adults $[14,15]$. In spite of that, the world literature lacks any information as concerns the role of $H$. pylori in childhood metabolic syndrome (childhood obesity and childhood diabetes).

H. pylori colonized the stomach since an immemorial time as if both the stomach and the bacterium used to live together in peace harmless to each other and hence $H$. pylori may be considered a natural bacterium. Assuming that $H$. pylori is a natural bacterium, it could travel from stomach to stomach via meals; therefore, existence of $H$. pylori in children starts trans-familial during early childhood. This suggestion is confirmed by the fact that $H$. pylori strain of children is often identical with that of their parents. Interestingly, children maintain the same strain genotype even after moving to a different environment $[9,10]$. The challenge lies in the emergence of antibiotic-resistant or abnormal behavior $H$. pylori strains, which is most probably due to the abuse of antibiotics or inefficiency of the current eradication strategies [16]. It would not be scientifically sound to cost the child's delicate physical structure or his immune system the drastic side-effects of repeated $H$. pylori antibiotic eradication therapies upon detection of $H$. pylori each time; revision of the current guidelines for the management of $H$. pylori may be needed [17].

H. pylori could migrate or get forced to migrate to the colon; it will continue producing ammonia for a reason or no reason, unopposed or buffered by any acidity, leading to accumulation of profuse toxic amounts of ammonia that could lead to toxic biological stress or toxic pancreatitis causing an onset of diabetes. If the abnormal behavior $H$. pylori strains in their abnormal colonic habitat could lead to adverse toxic effects in adults, certainly the child's delicate physical structure would be also severely affected by these strains $[9,10]$.

Dietary vinegar (acetic acid 5\%) has been recently demonstrated as dramatic, effective and decisive solution for all the challenges and medical problems related to $H$. pylori infection including eradication and re-infection. The cure rate of vinegar therapy reaches above $97 \%$ 
with negligible incidence of failure of treatment or recurrence of infection. The application of vinegar therapy in relief of symptoms and cure of $H$. pylori is simply based on a definite pathophysiologic principle that can offer wonderful promises to many patients [18]. The complex nutritional requirements of $H$. pylori are achieved through its unique pyruvate energy metabolism, which exhibits characteristic dislocation sites. These sites can be considered as targets that should attract any attempts to fight the organism [12]. As acetate is demonstrated as an end product among the metabolic pathway of $H$. pylori; this means that addition of acetic acid to the atmosphere around $H$. pylori could compromise the energy metabolism or interfere with the organism's respiratory chain metabolism. This suggestion is supported by the fact that the major routes of generation of energy for $H$. pylori are via pyruvate and the activity of the pyruvate dehydrogenase complex is controlled by the rules of product inhibition and feedback regulation. It is further supported by the observation that addition of pyruvate to different solid culture media was found to inhibit bacterial growth, and this inhibition was attributed to accumulation of acetate and formate $[12,18,19]$. As the matter includes interference with the energy metabolism and the respiratory chain of $H$. pylori; an immediate paralysis of the organism could be considered with dramatic relief of patient's symptoms. The fast immediate influence of acetic acid on $H$. pylori gives no chance for the organism to resist the treatment with vinegar or to mutate and develop resistant strains.

In this study, the effect of pyruvate, 20 times dilutions of dietary vinegar (acetic acid 6\%) and three times dilution of the senna purge preparation added to $H$. pylori solid culture media was studied. Addition of pyruvate demonstrated a delayed inhibitory effect on the motility of $H$. pylori, while addition of the diluted vinegar and the diluted senna leaves extract showed an immediate lethal influence on H. pylori.

\section{References}

1. Habib HS (2005) Frequency and clinical characteristics of ketoacidosis at onset of childhood type 1 diabetes mellitus in Northwest Saudi Arabia. Saudi Med J 26: 1936-1939.

2. Sabin MA, Shield JP (2008) Childhood obesity. Front Horm Res 36: 85-96.

3. De Ferranti SD, Osganian SK (2007) Epidemiology of paediatric metabolic syndrome and type 2 diabetes mellitus. Diab Vasc Dis Res 4: 285-296.
4. Al-Nozha MM, Al-Maatouq MA, Al-Mazrou YY, Al-Harthi SS, Arafah MR, et al. (2004) Diabetes mellitus in Saudi Arabia. Saudi Med J 25: 1603-1610.

5. Salardi S, Cacciari E, Menegatti M, Landi F, Mazzanti L, et al. (1999) Helicobacter pylori and type 1 diabetes mellitus in children. J Pediatr Gastroenterol Nutr 28: 307-309.

6. Dore MP, Bilotta M, Malaty HM, Pacifico A, Maioli M, et al. (2000) Diabetes mellitus and Helicobacter pylori infection. Nutrition 16: 407-410.

7. Baron S (2000) Baronas medical microbiology. Churchill Livingstone 4: 346.

8. Kountouras J, Zavos C, Chatzopoulos D (2005) A concept on the role of Helicobacter pylori infection in autoimmune pancreatitis. J Cell Mol Med 9: 196-207.

9. Farinha P, Gascoyne RD (2005) Helicobacter pylori and MALT lymphoma. Gastroenterology 128: 1579-1605.

10. Nasrat SAM, Randa MN, Nasrat MN (2015) The dramatic spread of diabetes mellitus worldwide and influence of Helicobacter pylori. General Med 3: 159-162.

11. Bulut Y, Agacayak A, Karlidag T, Toraman ZA, Yilmaz M (2006) Association of cagA+ Helicobacter pylori with adenotonsillar hypertrophy. Tohoku J Exp Med 209: 229-233.

12. Kulaylat NA, Narchi H (2001) Clinical picture of childhood type 1 diabetes mellitus in the Eastern Province of Saudi Arabia. Pediatr Diabetes 2: 43-47.

13. Salman H, Abanamy A, Ghassan B, Khalil M (1991) Childhood diabetes in Saudi Arabia. Diabet Med 8: 176-178.

14. Nabipour I, Vahdat K, Jafari SM (2006) The association of metabolic syndrome and Chlamydia pneumoniae, Helicobacter pylori, cytomegalovirus, and herpes simplex type 1: the Persian Gulf Healthy Heart Study. Cardiovasc Diabetol 1: 5: 25.

15. Longo-Mbenza B, Nkondi Nsenga J, Vangu Ngoma D (2007) Prevention of metabolic syndrome insulin resistance and the atherosclerotic diseases in Africans infected by Helicobacter pylori infection and treated by antibiotics. Int J Cardiol 121: 229-238.

16. Ge Z (2002) Potential of fumarate reductase as a novel therapeutic target in Helicobacter pylori infection. Expert Opin Ther Targets 6: 135-146.

17. Gasbarrini A, Franceschi F (2005) Does H. Pylori infection play a role in idiopathic thrombocytopenic purpura and in other autoimmune diseases? Am J Gastroenterol 100: 1271-1273.

18. Nasrat AM (2006) A new approach for the hematologic challenges of Helicobacter pylori infection in children. The International Symposium of the Egyptian Society of Pediatric Hematology/Oncology

19. Cirak MY, Ozdek A, Yilmaz D, Bayiz U, Samim E, et al. (2003) Detection of Helicobacter pylori and its CagA gene in tonsil and adenoid tissues by PCR. Arch Otolaryngol Head Neck Surg 129: 1225-1229. 